\title{
Adaptive Inter-Area Oscillation Damping Controller for Multi-Machine Power Systems
}

\author{
Ke Tang and Ganesh K. Venayagamoorthy \\ Real-Time Power and Intelligent Systems Laboratory, Holcombe Department of Electrical and Computer Engineering \\ Clemson University, Clemson, SC 29634, United States
}

\begin{abstract}
The interconnection of multiple generation units in a power system can lead to inter-area oscillations that generally occur under critical operating conditions. To damp these oscillations, supplementary control can be provided by a wide area control (WAC) system using remote measurements from the power system. Wide area controllers designed with traditional approaches are non-adaptive and thus their control policy may not be optimal to nonlinear operation conditions and disturbances. In this paper, the concept of an artificial immune system (AIS) is applied in the development of an innate and adaptive controller to handle known, unknown and random disturbances in a power system. Furthermore, with synchronous generator coherency grouping, remote virtual generator measurements are used to generate supplementary control signals to a synchronous generator that is identified to have maximum controllability on a power system. Real-time simulation and frequency analysis results show the superior performance of AIS-based controller in damping inter-area oscillations for different power system conditions and disturbances.
\end{abstract}

Keywords: Adaptive and innate control, artificial immune system (AIS), damping control, electric power system, remote measurements, virtual generator, wide area control

\section{Introduction}

Oscillations are frequently observed in a power system operating under critical conditions as due to lack of sufficient damping torque [1]. Modal analysis using remote measurements reveals the presence of local, intra-area and inter-area modes and their respective damping ratios in an interconnected power system. Conventional power system stabilizers (PSSs) make use of feedback signals that are obtained locally such as speed deviation or active power output of a generator applied to provide supplementary control signal to the automatic voltage regulator (AVR). However, since the local measurements lack observability of inter-area modes, PSSs are only effective for damping local and intra-area modes [2]. In contrast, remote measurements used by wide area control (WAC) enhances control efficacy for the inter-area modes. In [3], inter-area oscillations are damped by static VAR compensator based on optimal measurement signal selection. In [4], WAC is 
implemented through phase compensation. An earlier study that shows a virtual generator based power system stabilizer (VGPSS) for damping of inter-area oscillation modes is reported in [5] by the authors. This VG-PSS uses remotely measured virtual generator speeds for supplementary damping control at the generator of maximum controllability. These abovementioned controllers have fixed parameters. However, it is desired that the parameters self-tune online so that the controllers are adaptive to various changing conditions that affect control effectiveness and system performance. In [6], a conventional multiple-model adaptive control scheme is applied to form a control signal from a bank of controllers; yet the number of operating conditions corresponding to each pre-designed controller is limited. A neural-network based adaptive critic control scheme for WAC is suggested in [7]; however, the update of neural networks parameters is offline, thus the parameters of this controller remain unchanged during the real-time operation of the power system. In [8], artificial immune system (AIS) is introduced for adaptive excitation control of generators in an electric ship to handle high energy demand loads such as pulsed loads.

In this paper, the concept of AIS is applied for the development of wide area signals based adaptive damping controller for inter-area oscillations. The wide area signals are derived from virtual generators formed by coherency grouping. Each coherent group is modeled by a virtual generator. Based on an optimal (innate) controller with best tuned parameters developed in [5], the AIS's adaptive strategy further modifies the control policy by temporarily adjusting controller parameters for unseen and abnormal disturbances. This is the second aspect of the AIS concept: an adaptive immune controller. Compared to a fixed parameter based damping controller, the AIS-based control is more agile and can stabilize a power system under various operating conditions and disturbances due to its innate and adaptive immune properties. By modeling biological immune systems, AIS adaptively and interactively generates feedback control signals to restore a system to its steady state. During the development of the optimal controller, generators in the power system are assumed to be oscillating in fixed coherent groups. However, it is known that a variation of operating conditions could lead to different coherent groups. For instance, generators near the fault site tend to lose oscillatory coherency [7]. Thus, the performance of the innate controller is degraded. Besides, since the oscillations occur in multiple frequencies during transients, a controller with fixed parameters may not be robust enough to handle all possible power system dynamic behavior for several operating conditions and disturbances. Fortunately, the impact of new and/or changing coherent groups and multiple modes can be minimized by adaptive nature of the AIS. The AIS controller parameters are only temporarily changed in response to perturbations caused by changes in operating conditions and disturbances. Post-disturbances or reaching equilibrium states, the controller parameters are restored to its optimal values for normal operating conditions.

The rest of the paper is organized as follows: In Section II, the inter-area oscillation in an interconnected electrical power system is briefly discussed. Section III presents an AIS structure and describes its mechanism for feedback and adaptive 
control. In Section IV, AIS is applied to an electrical power system to damp the inter-area oscillations. In Section V typical results using a real-time digital simulator (RTDS) and frequency response analysis are presented. Finally, conclusions are given in Section VI.

\section{Inter-Area Oscillations in Electrical Power Systems}

In an interconnected electrical power system, oscillatory behavior is inherent in the transient response to disturbances and changes in operating conditions such as generation and load variations. The oscillations manifest themselves, primarily, in generator rotor speeds and tie-line power flows, causing loss of generator synchronism, tripping of generators and loads, and furthermore islanding section(s) of power system. Generally, there are multi-modal oscillations that superimpose and interact with each other. Of all the modes, the most severe consequences may arise from inter-area modes caused by multi-areas in a power system swinging against each other, typically in the frequency range of $0.2 \mathrm{~Hz}-0.8 \mathrm{~Hz}$ [7].

The inter-area oscillations are caused by many factors that lead to decreased damping torque. The negative damping torque induced by AVRs of generators, the lack of active power transmission capacity linking two power system areas, as well as the nonlinear dynamic interactions between oscillation modes are all possible causes.

Linear differential equations based modeling of power systems can be used to study inter-area oscillations [10]. A linearized model around a nominal operating condition can be expressed using (1). The system matrices in (1) can be identified heuristically with a Stochastic Subspace Identification (SSI) algorithm by injection of Pseudo-Random Binary Signals (PRBSs) at AVRs of each generator and measuring corresponding speed deviation responses [11, 12].

$$
\begin{aligned}
& \dot{x}=A x+B u \\
& y=C x+D u
\end{aligned}
$$

Based on this state space representation, modal analysis can be carried out to determine the frequency and damping ratios of oscillations present [13]. Inter-area oscillation impacts large geological areas. The varying generation and load conditions lead to power flow change. For example, scheduled active power transaction in a deregulated market now varies in specific transmission lines. Network topology changes may result by contingencies due to poor damping. Besides, intermittent renewable energy generations bring stochastic change to the system power flow distribution. All these factors add to the difficulty in the development of an optimal damping controller that can be universally applied. It is desired that the controller is adaptive to accommodate the nonlinear, time-varying and stochastic properties of an interconnected power system.

It is observed that electrically adjacent generators tend to oscillate coherently due to the convenience of inter-machine active power transfer. The coherency is reflected by the proximity among speed deviation responses of a generator's cluster. Thus, redundancy can be reduced using a Virtual Generator (VG) as a mathematical equivalent of coherent generators, as 
expressed in (2), where $H$ signifies the inertia constant of a generator and the subscript $j$ signifies the index of a generator within the group. Detailed implementation of system identification, model analysis and generator coherency were elaborated in [5].

$$
\omega_{e q}=\left(\sum_{j=1}^{N} H_{j} \omega_{j}\right) /\left(\sum_{j=1}^{N} H_{j}\right)
$$

Speed deviation from VGs instead of single generators can be used as an input to a damping controller. Unfortunately, generator coherency is subject to changes due to variation of operating conditions as well as contingency types and locations. This may not be best handled by a VG-based damping controller with fixed parameters; instead, an adaptive controller is needed to handle the fast changing dynamics of a power system.

\section{Artificial Immune System}

The defensiveness of a biological immune system relies on the presence of innate and adaptive immunity. As an analogy to a biological immune system that is resistant to antigen incursion, AIS can provide enhanced power system stability by improving controller adaptiveness to disturbances and contingencies. To maximize control effectiveness, an AIS based adaptive controller is introduced in this paper. In the application of AIS, the innate immunity is realized through optimal parameter configuration, and the adaptive immunity is realized through adaptive change of controller parameters, as elaborated in the following subsections.

\subsection{Innate Immunity}

In the biological immune system, innate immunity refers to the ability of living body (such as human) to provide the primary defense reactions against incurring antigens by generating neutrophils (such as blood cells) to identify and destroy the antigens. The antigens are bound and engulfed by macrophages before being further demolished by neutrophils like white blood cells. The control mechanism in innate immunity is the ability to generate a control signal to maintain stability in response to the measured perturbation from equilibrium. This response behavior can be achieved by an optimal action and can be compared to a control system with fixed parameters.

\subsection{Adaptive Immunity}

In the biological immune system, the dendritic cells that bind antigens can be further evolved into antigen presenting cells (APCs), B cells and T cells. Based on this, a more sophisticated feedback law mechanism to provide adaptive immunity is present. When an antigen is detected in a living body, APC is generated that leads to the production of helper T cells. The 
helper $\mathrm{T}$ cells further activate $\mathrm{B}$ cells and killer $\mathrm{T}$ cells that swallow and annihilate the antigen. The suppressor $\mathrm{T}$ cells function to hinder the activity of all other cells activated by helper T cells as illustrated in Fig. 1 [14].

A feedback mechanism adaptively regulates the activity of cells. The presence of invading antigens leads to more activated helper $\mathrm{T}$ cells that significantly increase the amount of killer $\mathrm{T}$ cells and $\mathrm{B}$ cells. Over time when the antigens diminish and helper $\mathrm{T}$ cells decrease in activity, both killer $\mathrm{T}$ cells and $\mathrm{B}$ cells are inhibited by suppressor $\mathrm{T}$ cells that have been already generated. Ultimately, the antigens are completely diminished, and killer T cells and B cells are deactivated.

Fig. 1. A biological immune system

In an AIS-based controlled system, as soon as the system moves away from its steady state, the controller parameters are regulated over time to restore the system to its steady state as quickly as possible. The deviation of the controlled system from its steady state is analogous to the antigen, while the amount of $\mathrm{T}$ cells and B cells is analogous to the regulation of controller parameters. When steady state has been achieved, the controller parameters return to their innate values.

The nominal value for each of the $N$ controller parameters is denoted as $p_{i, \text { standard }}(i=1,2, \ldots N)$. The deviation of parameter at the moment of $k$ is denoted as $\Delta p_{i}(k)$ as indicated in (3). The mechanism of parameter updating process using AIS is shown in Fig. 2.

$$
p_{i}(k)=p_{i, \text { standard }}+\Delta p_{i}(k)
$$

where

$$
\Delta p_{i}(k)=T H_{i}(k)-T S_{i}(k)
$$

Fig. 2. The schematic of the proposed AIS based control for a dynamic system.

It is comprised of two competing terms. The first term is analogous to helper T cells that activates the control effect; and is directly proportional to $\Delta z$ as expressed in (5), where $m_{i 1}$ is a stimulation factor to the gain of helper T cells.

$$
T H_{i}(k)=m_{i 1} \Delta z(k)
$$

The second term in (4) corresponds to suppressor $\mathrm{T}$ cells that diminish the control effect. It is inversely related to the amplitude of the changing rate at $\Delta z . T S_{i}(k)$ can be expressed as (6), in which $m_{i 2}$ is an inhibition factor related to the gain of suppressor T cells. According to (6), the faster declining rate of $\Delta z$ leads to the larger value of $T S_{i}(k)$, which counteracts the parameter change brought by $T H_{i}(k)$. The output of division block in Fig. 2 is reset to zero when steady state is reached.

$$
T S_{i}(k)=m_{i 2} \Delta z \exp \left\{-\left(\frac{\Delta z(k)}{\Delta z(k-1)}\right)^{2}\right\}
$$


Equations (3)-(6) can also be applied for the update of any other controller parameters, so that all of them will be regulated dynamically by AIS. Since two extra AIS parameters $\left(m_{i 1}, m_{i 2}\right)$ are required for the update of one controller parameter $p_{i}$, totally $2 N$ AIS parameters are introduced in addition to the ten parameters in the innate controller (described later).

The adaptive immunity brings about robustness against changes of operating conditions, leading to enhanced system stability. This is achieved by optimal tuning of innate and adaptive controller parameters, as elaborated in the following section.

\section{4. $\quad$ AIS Based Oscillation Damping Controller}

In this section, the development of an AIS-based adaptive controller for damping inter-area oscillation in an interconnected power system is described. The flowchart for the development is given in Fig. 3 with details elaborated in the following subsections.

Fig. 3. Flowchart illustrating the development of an AIS based damping controller.

The following subsections describe the test power system used in this study, as well as the innate and adaptive damping controller. The adaptive control behavior is only a transient behavior to enhance controller performance.

\subsection{Test Electrical Power System}

To illustrate the effectiveness of the AIS oscillation damping controller, an IEEE 68-bus 16-machine electrical power system is used that consists New England (NE), New York (NY) and other systems modeled as generators (G14-G16), as shown in Fig. 4. NE and NY are linked with three major corridors (Bus 8-Bus 9, Bus 1-Bus 27, Bus 1-Bus 2). Bus1-Bus2 is the most crucial connection with the largest transmission capacity.

Fig. 4. IEEE 68-bus system with five coherent groups.

Five coherent groups are identified using hierarchical clustering, as indicated in Table I [15]. Fig. 5 is an indication of speed deviations of all the 16 generators following a contingency, with each coherent group marked by a distinct color. Through modal analysis, oscillations at $0.22 \mathrm{~Hz}, 0.42 \mathrm{~Hz}$ and $0.54 \mathrm{~Hz}$ can be observed to be the most prominent inter-area oscillation modes [16]. It is shown in Fig. 6 that the power transfer from NE to NY has an impact on the damping ratios of various oscillation modes. The damping ratio decrease of the $0.22 \mathrm{~Hz}$ mode is especially obvious as the inter-area power transfer increases. Moreover, when a critical transmission line (such as Bus1-Bus2) linking the two areas is tripped following a contingency, a weaker system results and the oscillation may be further deteriorated and lead to loss of stability.

As indicated in Fig. 7, modal analysis also has shown that G9 has the highest controllability for the oscillation mode of $0.22 \mathrm{~Hz}$ under critical operating conditions. 
Traditional local PSS makes use of local generator speed deviations to generate a supplementary control signal, which is superimposed on the voltage reference at the AVR of local generator. In this study, local PSSs are designed based on $\mathrm{H}_{\infty}$ based robust control approaches [17], and are installed on G1-G12 to maintain power system stability. However, since poorly damped oscillations occur under critical operating conditions, robust wide-area damping controllers are required.

An innate controller scheme that utilizes the speed deviations of all VGs for supplementary excitation control is suggested, as indicated in Fig. 8. According to the clustering result in Table I, the five VG speed deviations are taken as inputs for the innate controller; their weighted sum is fed as an input to a washout filter followed by a two-stage lead-lag compensator, and finally a magnitude-limiter to serve as the supplementary control signal at the AVR of G9. Thus by taking into consideration the speed responses of all virtual generators, oscillations in different modes have been used in deciding the control signal. Ten parameters are present in the innate controller: the weights of VG speed deviations, the lead-lag gain and time constants.

Fig. 5. Generators oscillating in coherent groups.

Fig. 6. Damping ratio change with respect to active power transfer from NE to NY.

Fig. 7. The controllability factors for all generators for $0.22 \mathrm{~Hz}$ mode.

Table I Coherent grouping of generators

The ten parameters of the innate controller are determined through particle swarm optimization (PSO) that heuristically minimizes the speed deviation of all generators during a 50ms three-phase fault at Bus 8 . In this study, considering the speed deviation responses from all generators, the cost function adopted in PSO is expressed as (7),

$$
J=-\alpha \min \left\{\xi_{f}, \xi_{0}\right\}+\sum_{j=1}^{16} \sum_{k=1}^{N}\left(\Delta \omega_{j}(k)\right)^{2}
$$

where $\alpha$ is a positive constant, $\xi_{f}$ is the damping ratio at $0.22 \mathrm{~Hz}, \xi_{0}$ is a threshold of $10 \%$ that represents a satisfactory damping ratio, and $\Delta \omega_{j}(k)$ is the speed deviation response of generator $j$ at the moment of $k$. In PSO, each set of system parameters is represented as a particle. The $i^{\text {th }}$ particle is updated in terms of (8) and (9),

$$
\begin{gathered}
\Delta v_{i, k}=\eta_{0} v_{i, k-1}+\eta_{1}\left(p_{i, l b e s t}-p_{i, k-1}\right)+\eta_{2}\left(p_{g b e s t}-p_{i, k-1}\right) \\
p_{i, k+1}=p_{i, k}+\Delta v_{i, k}
\end{gathered}
$$

Fig. 8. Diagram of the proposed innate controller scheme.

where $p_{i}$ signifies one set of parameters, while $\Delta v_{i}$ signifies the changing rate of $p_{i}$. 
In this approach, unlike $\mathrm{H}_{\infty}$ based controller design that yields five high-order transfer functions linking the five inputs and the output, the proposed innate controller has a simple mathematical expression with only ten parameters, facilitating further approaches for adaptive parameter adjustments as elaborated in subsection C. The tuned parameters are shown in Table A.II in the Appendix. In the practice, this innate controller is installed at G9. The method to compensate the time delay induced by data communication was successfully implemented in earlier study in [5]. Recent researches also see the practice of using neural network based predictions to overcome the time delay [18].

\subsection{Adaptive Damping Controller}

Based on the innate controller proposed in Section IV.B, an AIS-based adaptive damping controller is developed as shown in Fig. 9. For each of the ten innate controller parameters, two extra AIS-parameters are required. Thus, there are totally 20 AIS-parameters $\left(m_{1} \sim m_{20}\right)$ to be determined. The local speed deviation $\Delta \omega_{9}$ is used as the input for the AIS, thus the time delay is not considered by the adaptive damping controller.

The stimulation and inhibition factors are determined through PSO approach, with the cost function expressed in (11),

$$
J=\sum_{i=1}^{16} \sum_{j=1}^{N}\left(\Delta \omega_{i}(j)\right)^{2}
$$

with all symbols holding the same meaning as previously defined for the second term in (7). The tuning with PSO is based on same equations of (8) and (9). However, since the innate controller has already increased damping torque for the most severe oscillation mode, a different cost function is used now for the development of adaptive controller. Unlike the cost function in (7) that focuses on one oscillation mode, (11) only considers the time domain speed deviation responses. According to Parseval's theorem [19], the presence of any oscillation mode adds a positive value to $J$. Thus, the optimization will enhance the damping for various oscillation modes. The tuning process is carried out under simulation of 50ms three-phase faults at Bus 31. The tuned stimulation and inhibition factors are indicated in Table A.III in the Appendix.

The coordination of these two controllers is achieved by the consecutive tuning of innate and adaptive controller. In response to oscillations following disturbances, with parameters adjusted by the adaptive controller in the real time, the innate controller is able to provide a near-optimal supplementary control signal at any moment, making the power system survivable under more critical operation conditions.

Fig. 9. Schematic diagram of the AIS based controller.

\subsection{Tuning Platform}


The implementation of parameter-tuning for the innate and adaptive controller is carried out with using a hybrid platform consisting of the real-time digital simulator (RTDS) and MATLAB, as shown in Fig. 10.

During real-time simulation of a power system with change in operating condition(s) or disturbance, the AIS is implemented with a C-based program embedded as a user-built RTDS component. The time delay of $100 \mathrm{~ms}$ is assumed for the $\mathrm{z}^{-1}$ block (Fig. 9). The PSO algorithm is implemented in MATLAB, which interfaces with the RTDS through a C-like script language. Prior to simulation of a disturbance on the power system, RTDS reads and updates the innate or adaptive controller parameters obtained by the PSO algorithm. There are two stages in the determination of the respective parameters of the AIS controller. In the first stage, innate controller parameters are optimized by PSO tuning. Thereafter, with fixed optimal innate controller parameters, the optimal parameters of the adaptive AIS controller are determined.

Fig. 10. Development of the AIS controller.

(This figure shows the interface between the RTDS and MATLAB platforms.)

\section{Performance Evaluation of AIS controller}

Post development of the innate and adaptive controller, the effectiveness of the adaptive function of the AIS controller with optimized parameters is verified through simulation carried out on the RTDS. Like in the development of the AIS controller, the AIS controller is implemented with a C-based program embedded as a user-built RTDS component for online operation. A diagram for implementation of online AIS controller performance evaluation is shown in Fig. 11.

Fig. 11. Implementation of the AIS controller for online performance evaluation

(All components of power systems, innate controller and AIS are simulated on RTDS)

Three cases are studied corresponding to three different operating conditions as well as contingencies, with different fault site, power generation and loading conditions. The active power of each generator under these operating conditions are documented in the Table A.I in the Appendix. Compared with the study cases in [5], the active power transfer from NE to NY is higher, thus the transmission lines linking these two subsystems are subject to heavier power flows, leading to easier loss of oscillatory instability.

Case I: The power system is operating under Operating Condition 1 (OC1), then a 50ms three-phase fault happened at Bus 2, causing the transmission line connecting Bus 1 and Bus 2 to be tripped. The speed responses of some selected generators are shown in Fig. 12 when local PSSs are installed in G1-G12. Without installation of innate controller, the oscillation amplitude increases with the time until loss of synchronism. The innate controller is effective to maintain stability, while AIS-based adaptive controller improves the damping effectiveness. A comparison of the damping ratio for each oscillation mode with and without the designed controller is shown in Table II. These damping ratios are calculated using Prony Analysis [16]. 
Fig. 12. Speed responses of selected generators with local PSSs installation under Case I.

Case II: With the transmission line from Bus 1 to Bus 2 already tripped, the system operating condition change to OC2, then a 100ms three-phase fault happened at Bus 8. The speed responses of some selected generators are shown in Fig. 13 when local PSSs are installed in G1-G12. With only the local PSSs, the power system quickly loses synchronism. With the installation of innate controller, the system is marginally stable. With both the innate and adaptive controller, the system quickly recovers to steady state. The comparison of the damping ratio for each oscillation mode with and without the designed controller is shown in Table II.

Fig. 13. Speed responses of selected generators with local PSSs installation under Case II.

Case III: System operating conditions change to OC3, then a 100ms three-phase fault happened at Bus 27. Compared with OC2, there is an increase of active power transfer from New England to New York, and the load at Bus 1 has increased by 100\%. The speed responses of some selected generators are shown in Fig. 14 with local PSSs installation on G1-G12. With only the innate controller, power system loses stability following the disturbances. However, the power system is stabilized with the installation of both innate and adaptive controller. The comparison of the damping ratio for each oscillation mode with and without the designed controller is shown in Table II.

The deviation of the innate controller parameters with respect to time under the three cases described above are shown in Fig. 15.

Fig. 14. Speed responses of selected generators with local PSSs installation under Case III.

Fig. 15. Deviation of parameters with time for all three case studies (Case I, II \& III).

Table II Damping ratio for different modes under Case I - III

\section{Conclusions}

This paper has proposed an AIS-based wide area adaptive damping controller that uses virtual generator speeds for supplementary control signal to mitigate inter-area oscillations in a power system. The control is carried out at the generator of maximum controllability identified through modal analysis. The optimal tuning of controller parameters provides innate immunity, while adaptive immunity is available by a mechanism to adjust controller response policy according to the measured disturbance. The parameters of innate and adaptive controllers are tuned by particle swarm optimization. As verified by realtime simulations, the proposed AIS based controller is able to stabilize the power system under different disturbances, and achieve better performance under various operating conditions. This is possible as a result of the adaptive function of the AIS 
controller that varies the optimal controller parameters during the disturbances and returns the parameter values to its default values (innate controller).

Future research can be in two directions. With the variations experienced and data collected over time as a result of the AIS's adaptive controller parameter function, an automatic strategy to migrate the optimality of the controller can be investigated. The second direction will be to integrate the application of online synchronous generators coherency grouping in the AIS controller by investigating an online methodology.

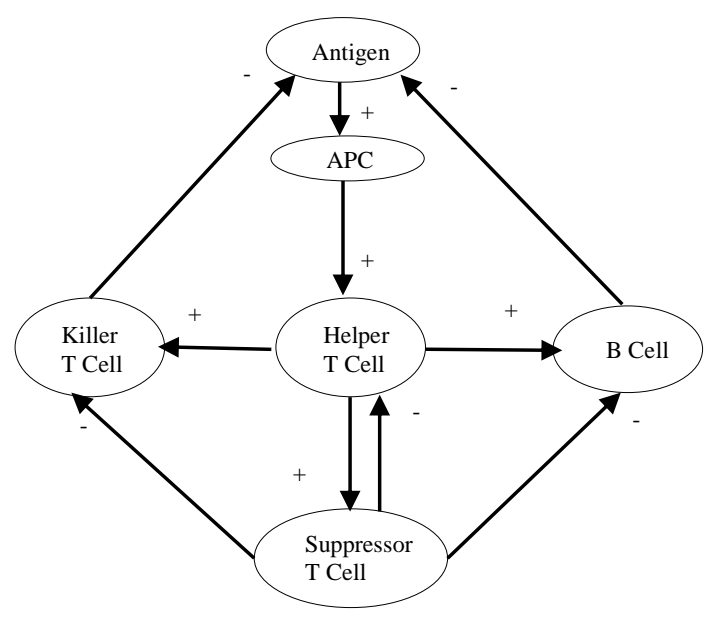

Fig. 1. A biological immune system 


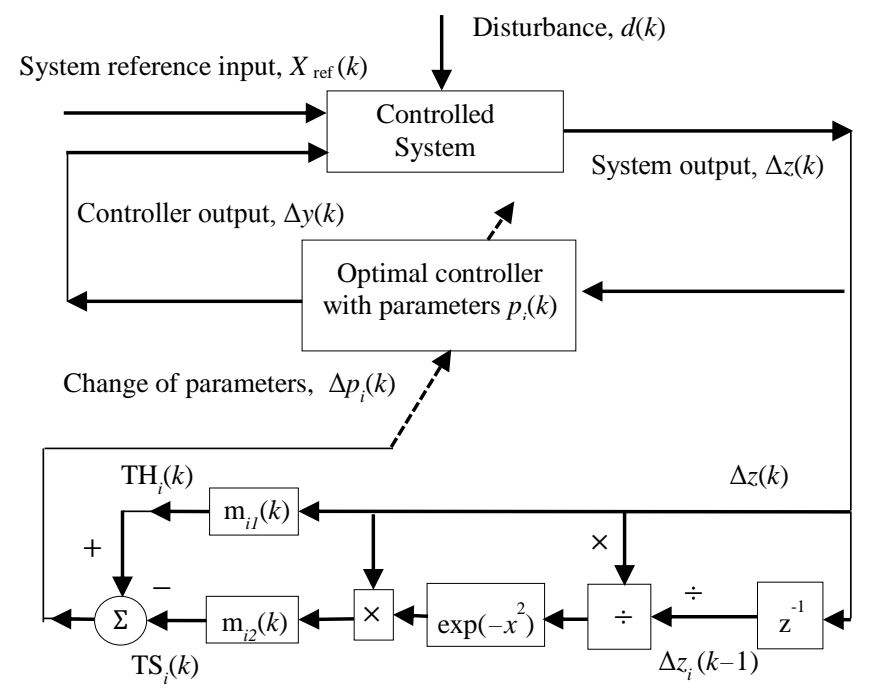

Fig. 2. The schematic of the proposed AIS based control for a dynamic system.

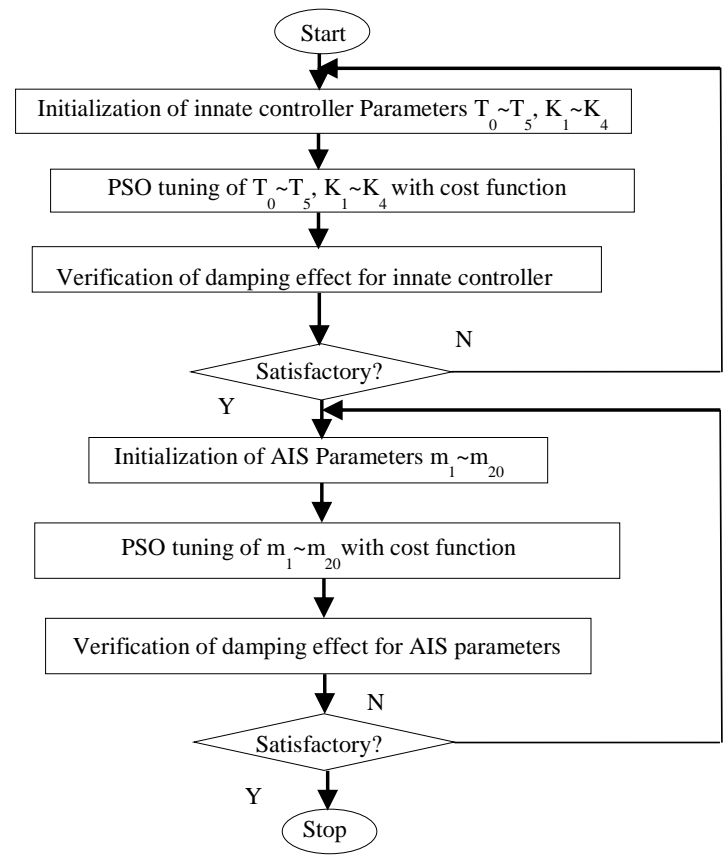

Fig. 3. Flowchart illustrating the development of an AIS based damping controller. 


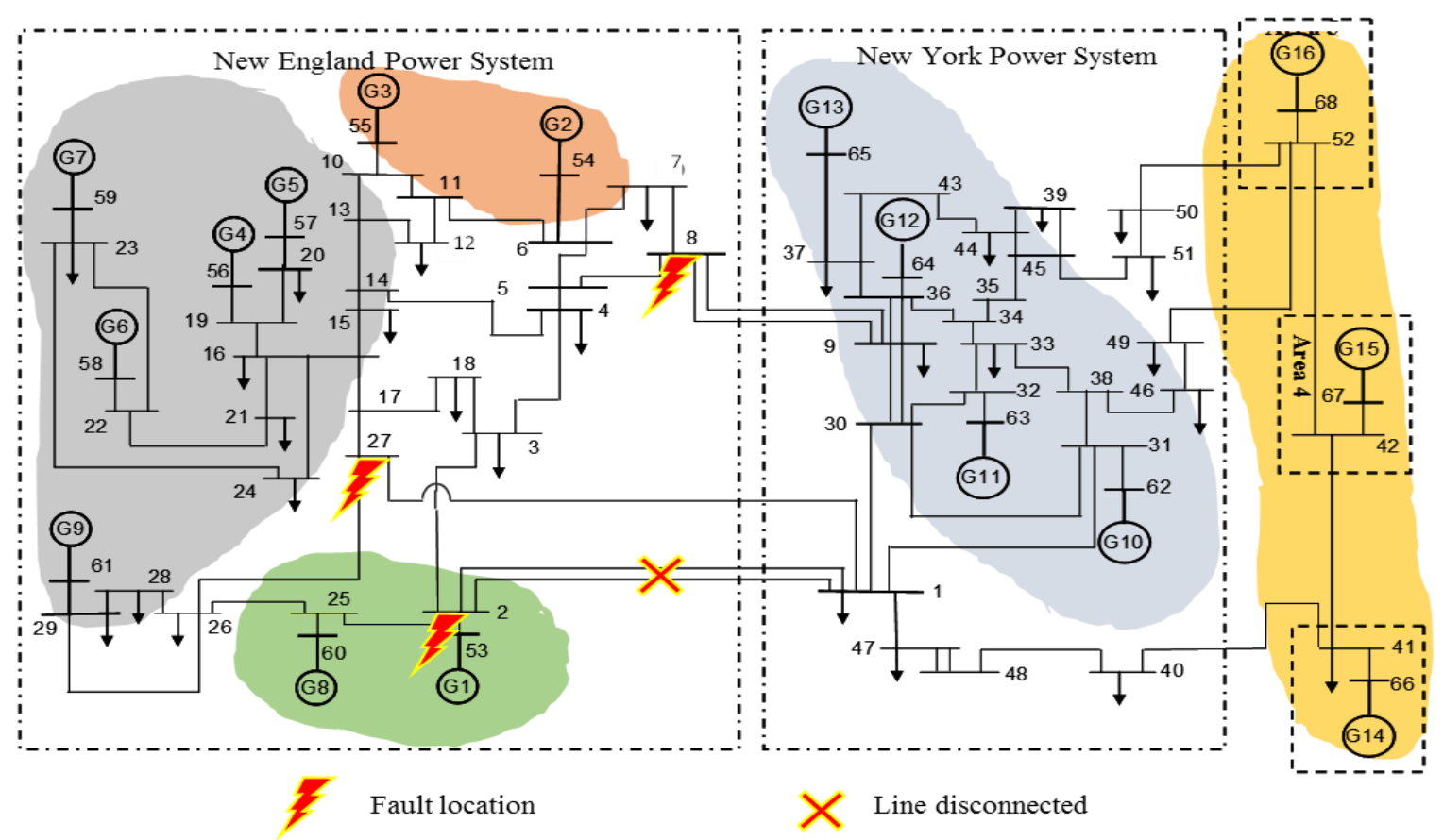

Fig. 4. IEEE 68-bus system with five coherent groups.

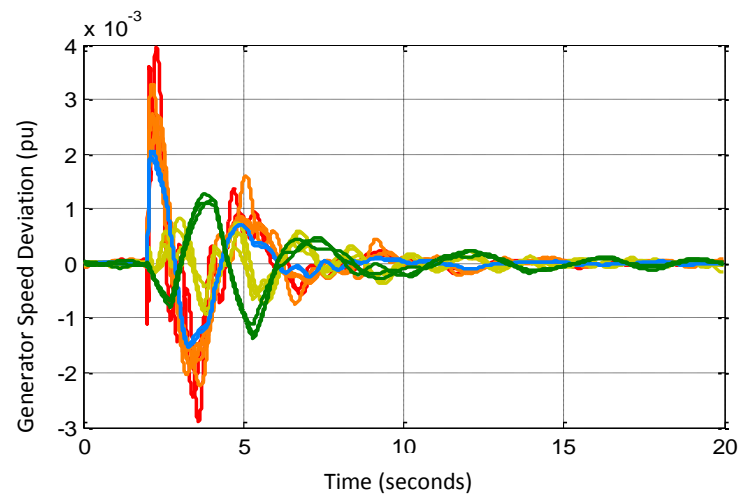

Fig. 5. Generators oscillating in coherent groups. 


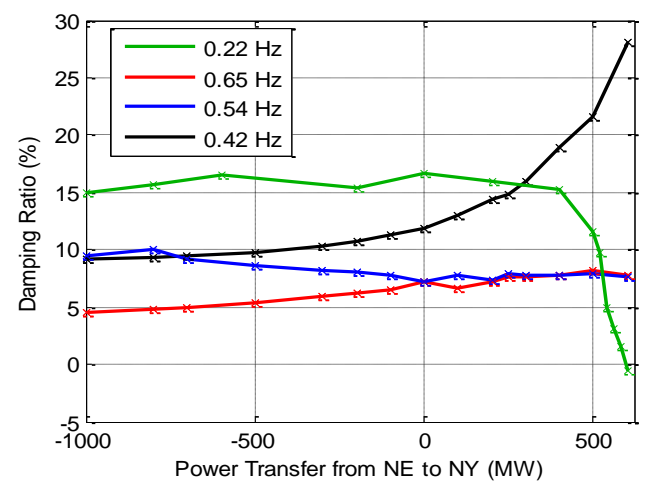

Fig. 6. Damping ratio change with respect to active power transfer from NE to NY.

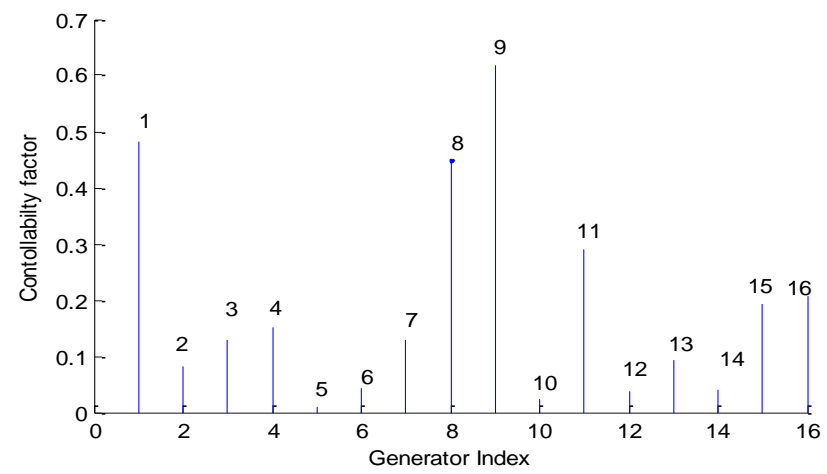

Fig. 7. The controllability factors for all generators for $0.22 \mathrm{~Hz}$ mode.

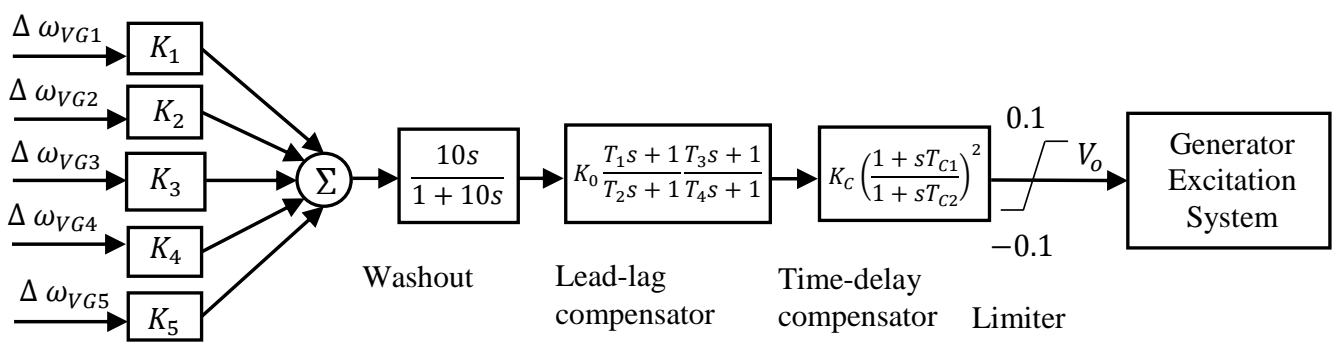

Fig. 8. Diagram of the proposed innate controller scheme. 


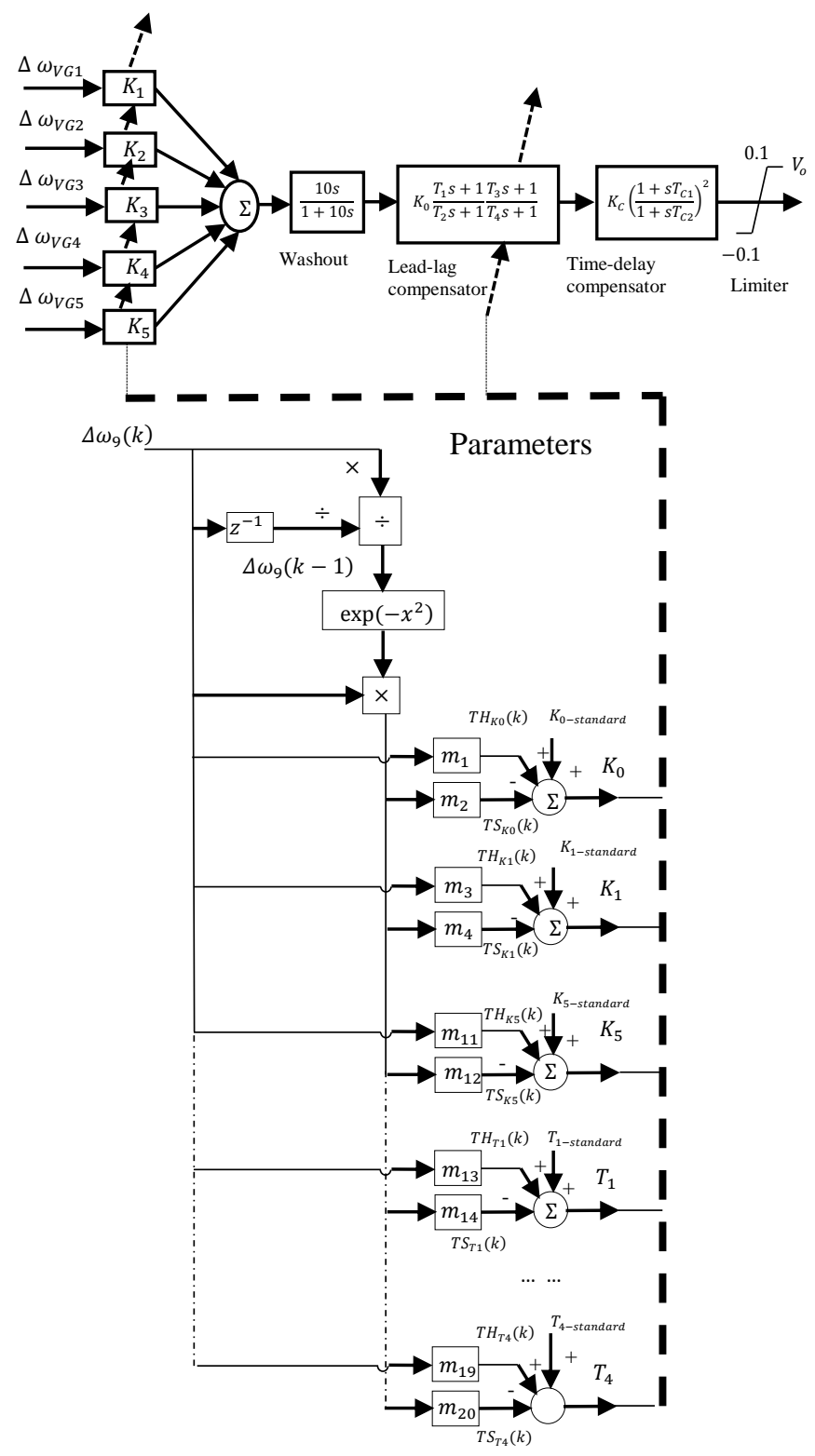

Fig. 9. Schematic diagram of the AIS based controller. 


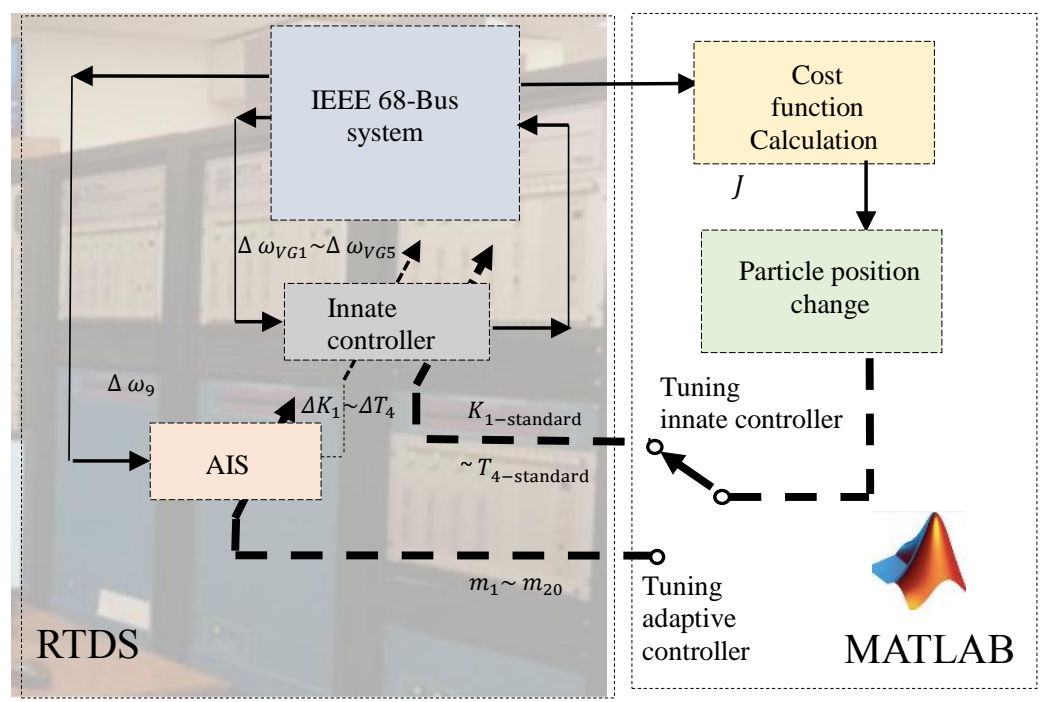

Fig. 10. Implementation of controller parameter configuration

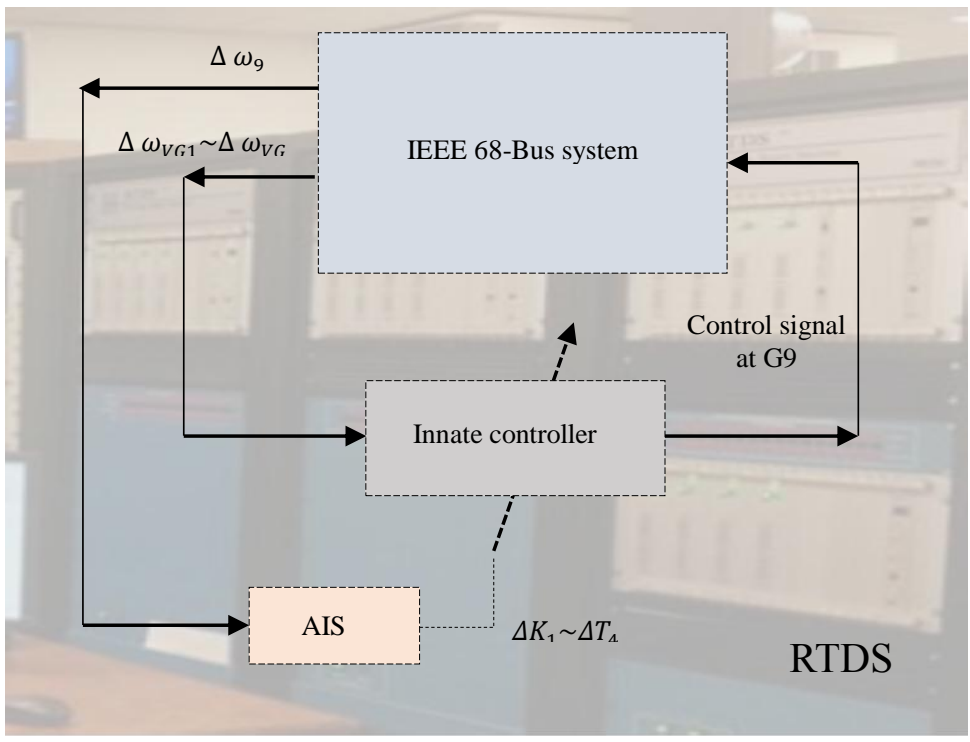

Fig. 11. Implementation of AIS controller for performance evaluation 

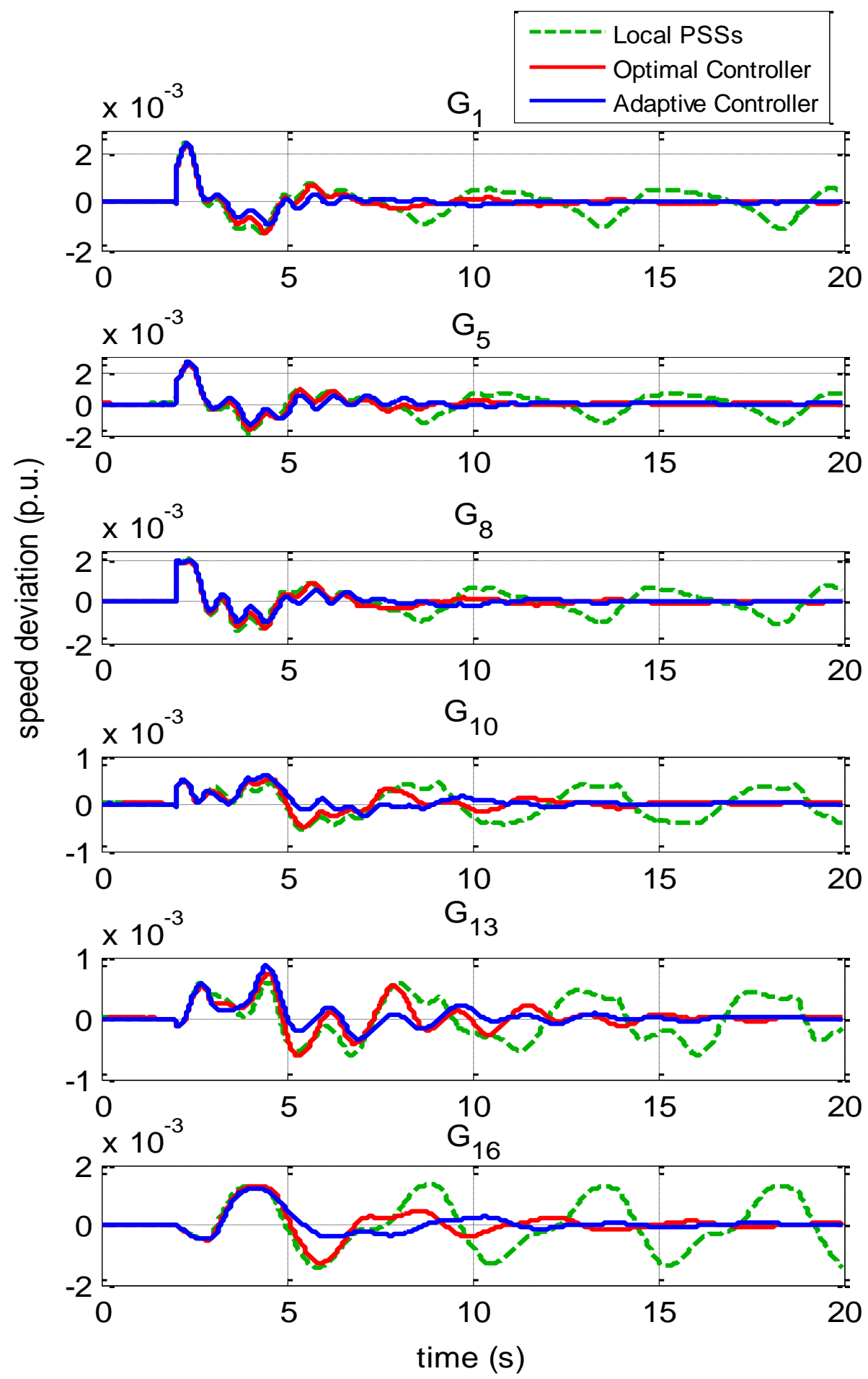

Fig. 12. Speed responses of selected generators with local PSSs installation under Case I. 


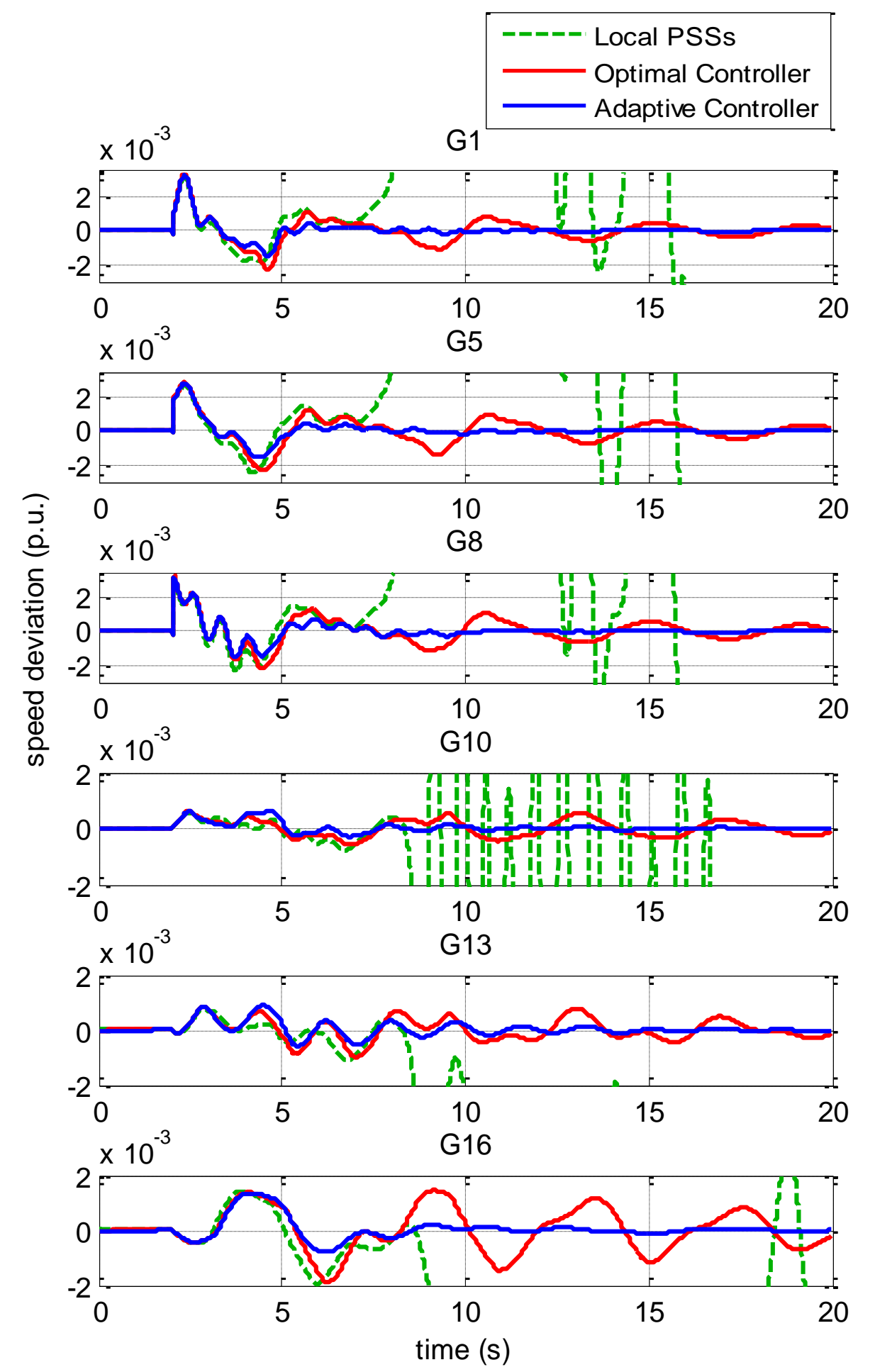

Fig. 13. Speed responses of selected generators with local PSSs installation under Case II. 


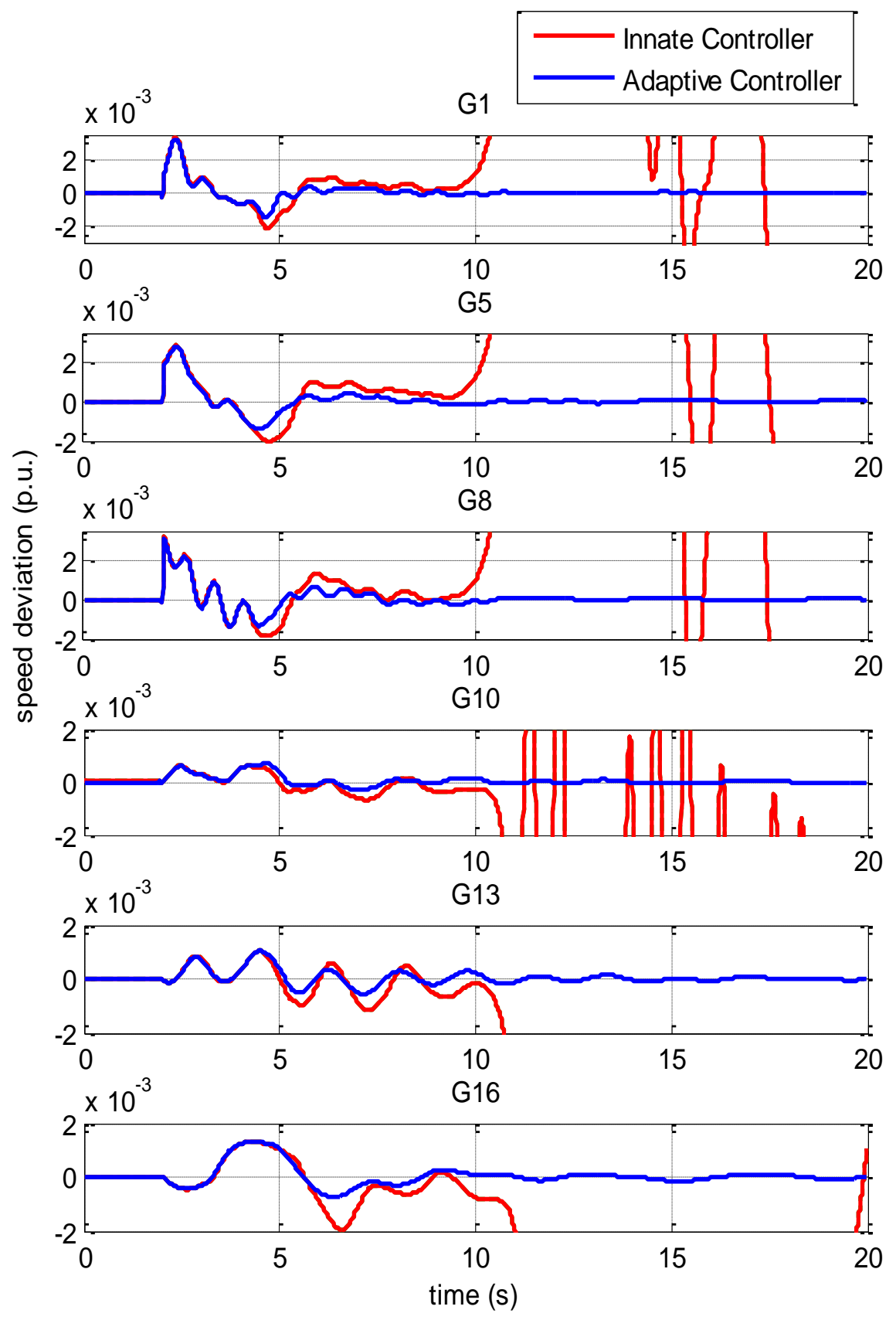

Fig. 14. Speed responses of selected generators with local PSSs installation under Case III. 

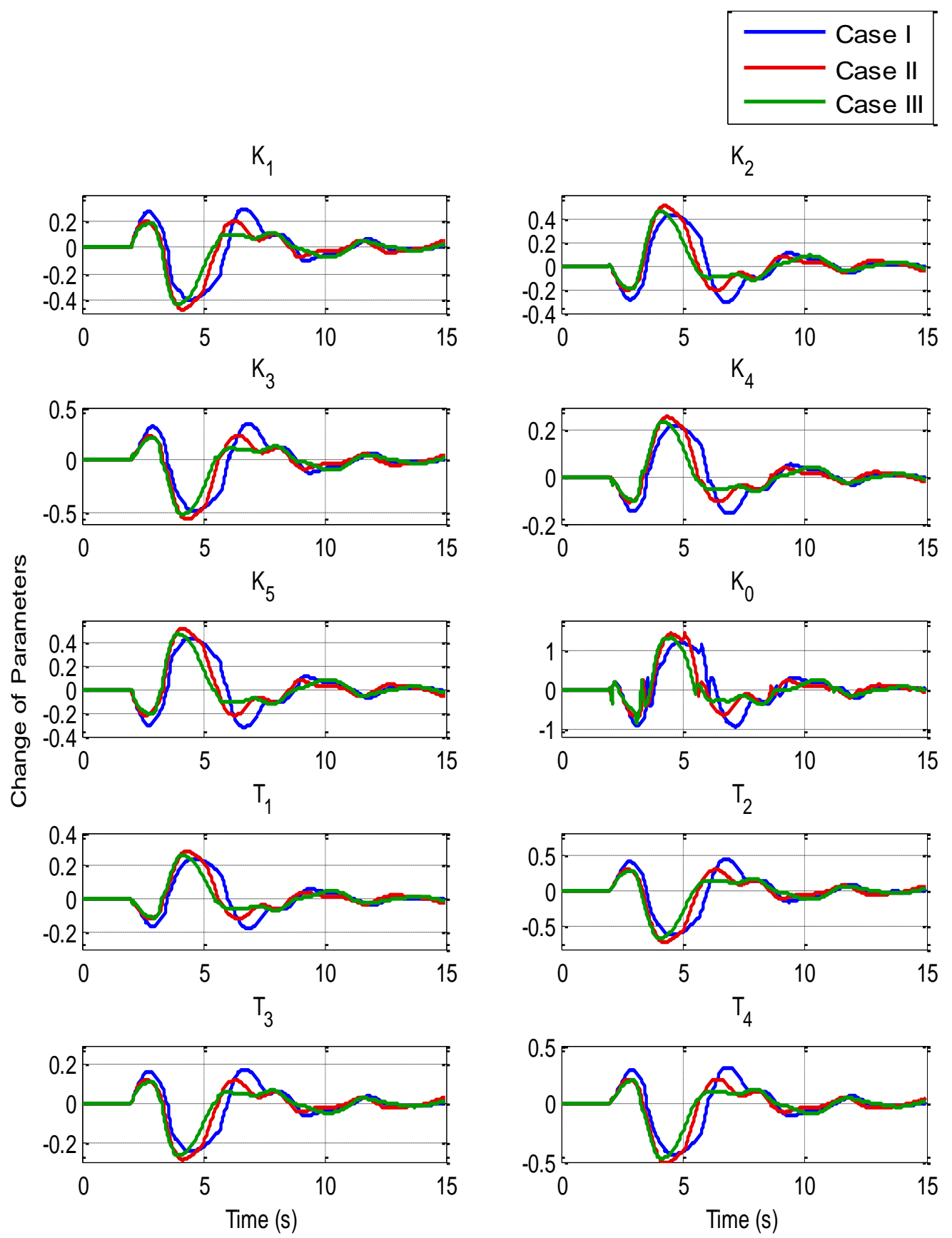

Fig. 15. Deviation of parameters with time for all three case studies (Cases I, II \& III). 
Table I Coherent grouping of generators

\begin{tabular}{cc}
\hline Group 1 & G1, G8 \\
\hline Group 2 & G2, G3 \\
\hline Group 3 & G4, G5, G6, G7, G9 \\
\hline Group 4 & G10, G11, G12, G13 \\
\hline Group 5 & G14, G15, G16 \\
\hline
\end{tabular}

Table II Damping ratio for different modes under Case I - III

\begin{tabular}{|c|c|c|c|c|c|}
\hline Cases & Cases & $0.22 \mathrm{~Hz}$ & $0.42 \mathrm{~Hz}$ & $0.54 \mathrm{~Hz}$ & $0.65 \mathrm{~Hz}$ \\
\hline \multirow{3}{*}{$\begin{array}{l}\text { Only with } \\
\text { local PSSs }\end{array}$} & Case I & $-2 \%$ & $12.6 \%$ & $7.3 \%$ & $6.9 \%$ \\
\hline & Case II & \multicolumn{4}{|c|}{ Unstable } \\
\hline & Case III & \multicolumn{4}{|c|}{ Unstable } \\
\hline \multirow{3}{*}{$\begin{array}{l}\text { With innate } \\
\text { controller }\end{array}$} & Case I & $9.1 \%$ & $15.2 \%$ & $11.2 \%$ & $12.4 \%$ \\
\hline & Case II & $3 \%$ & $14.1 \%$ & $10.2 \%$ & $11.9 \%$ \\
\hline & Case III & \multicolumn{4}{|c|}{ Unstable } \\
\hline \multirow{3}{*}{$\begin{array}{c}\text { With adaptive } \\
\text { controller }\end{array}$} & Case I & $14.6 \%$ & $17.1 \%$ & $13.5 \%$ & $13.1 \%$ \\
\hline & Case II & $15.6 \%$ & $16.1 \%$ & $14.5 \%$ & $13.6 \%$ \\
\hline & Case III & $14.7 \%$ & $16.3 \%$ & $13.9 \%$ & $14.4 \%$ \\
\hline
\end{tabular}




\section{Appendix}

Table A.I The reference of active power generation for all generators

\begin{tabular}{|c|c|c|c|c|}
\hline Generator & $\begin{array}{c}\text { Base Case } \\
(\mathrm{MW})\end{array}$ & $\begin{array}{l}\text { OC } 1 \\
(\mathrm{MW})\end{array}$ & $\begin{array}{l}\text { OC } 2 \\
(\mathrm{MW})\end{array}$ & $\begin{array}{l}\text { OC } 3 \\
\text { (MW) }\end{array}$ \\
\hline G1 & 250 & 279.4 & 281.1 & 283.3 \\
\hline G2 & 545 & 574.4 & 576.1 & 578.3 \\
\hline G3 & 650 & 679.4 & 681.1 & 683.3 \\
\hline G4 & 632 & 661.4 & 663.1 & 665.3 \\
\hline G5 & 505.2 & 534.6 & 536.3 & 538.5 \\
\hline G6 & 700 & 729.4 & 731.1 & 733.3 \\
\hline G7 & 560 & 589.4 & 591.1 & 593.3 \\
\hline G8 & 540 & 569.4 & 571.1 & 573.3 \\
\hline G9 & 800 & 829.4 & 831.1 & 833.3 \\
\hline G10 & 500 & 500 & 500 & 500 \\
\hline G11 & 1150 & 1150 & 1150 & 1150 \\
\hline G12 & 1350 & 1350 & 1350 & 1350 \\
\hline G13 & 3445 & 3180 & 3165 & 3595 \\
\hline G14 & 1785 & 1785 & 1785 & 1785 \\
\hline G15 & 1000 & 1000 & 1000 & 1000 \\
\hline G16 & 4000 & 4000 & 4000 & 4000 \\
\hline
\end{tabular}


Table A.II Parameters of the tuned innate controller

\begin{tabular}{cccc}
\hline Parameter & Value & Parameter & Value \\
\hline $\mathrm{K}_{1}$ & -0.2318 & $\mathrm{~K}_{0}$ & 19.2026 \\
\hline $\mathrm{K}_{2}$ & 0.0524 & $\mathrm{~T}_{1}$ & 0.4680 \\
\hline $\mathrm{K}_{3}$ & 0.8703 & $\mathrm{~T}_{2}$ & 0.7744 \\
\hline $\mathrm{K}_{4}$ & 0.5533 & $\mathrm{~T}_{3}$ & 6.8278 \\
\hline $\mathrm{K}_{5}$ & 0.9963 & $\mathrm{~T}_{4}$ & 2.7501 \\
\hline
\end{tabular}

Table A.III. Tuned stimulation and inhibition factors

\begin{tabular}{ccccccc}
\hline$m_{1}$ & $m_{2}$ & $m_{3}$ & $m_{4}$ & $m_{5}$ & $m_{6}$ & $m_{7}$ \\
\hline 0.4495 & -0.3506 & -0.3514 & 0.0081 & 0.2286 & 0.4323 & -0.0846 \\
\hline$m_{8}$ & $m_{9}$ & $m_{10}$ & $m_{11}$ & $m_{12}$ & $m_{13}$ & $m_{14}$ \\
\hline-0.2564 & -0.4868 & 0.3535 & 0.0894 & -0.5 & -0.2395 & -0.4265 \\
\hline$m_{15}$ & $m_{16}$ & $m_{17}$ & $m_{18}$ & $m_{19}$ & $m_{20}$ & \\
\hline 0.5 & -0.0305 & 0.2578 & -0.1623 & 0.2424 & 0.3076 & \\
\hline
\end{tabular}


References

[1] P. Sauer and M. Pai, Power System Dynamics and Stability. Upper Saddle River, NJ: Prentice-Hall, 1998.

[2] S. Ray, G. K. Venayagamoorthy, "Wide-area signal-based optimal neuro-controller for a UPFC," IEEE Transactions on Power Delivery, pp. 1597-1605, vol. 23, 2008.

[3] A. Heniche, I. Kamwa, "Assessment of two methods to select wide-area signals for power system damping control," IEEE Transactions on Power Systems, vol. 23, pp. 572-581, 2008.

[4] P. Zhang, D.Y. Yang, et al. , "Adaptive wide-area damping control scheme with stochastic subspace identification and signal time delay compensation," IET Generation, Transmission \& Distribution, vol. 6, pp. 844-852, 2011.

[5] K. Tang, G. K. Venayagamoorthy, "Damping inter-area oscillations using virtual generator based power system stabilizer", Electric Power Systems Research, Vol. 129, December 2015, pp. 126-141.

[6] B. Chaudhuri, R. Majumder, et al., "Application of multiple-model adaptive control strategy for robust damping of interarea oscillations in power system," IEEE Transactions on Control System Technology, vol. 12, pp. 727-736, 2004.

[7] D. Molina, G. K. Venayagamoorthy, et al., "Intelligent local area signals based damping of power system oscillations using virtual generators and approximate dynamic programming," IEEE Transactions on Smart Grid, pp. 498-508, 2013.

[8] C. Yan, G. K. Venayagamoorthy, K. Corzine, “AIS-based coordinated and adaptive control of generator excitation systems for an electric ship," IEEE Transactions on Industrial Electronics, vol. 59, pp. 3102-3112, 2012.

[9] P. Mitra, G. K. Venayagamoorthy, "An adaptive control strategy for DSTATCOM applications in an electrical ship power system," IEEE Transactions on Power Electronics, vol. 25, pp. 95-104, 2009.

[10] P. Tabuada, S. Y. Caliskan, "Compositional transient stability analysis of multimachine power networks," IEEE Transactions on Control of Network Systems, pp. 4-14, vol. 1, 2014.

[11] N. J. Bershad, J. C. M. Bermudez, J. Y. Tourneret, "Stochastic analysis of the LMS algorithm for system identification with subspace inputs," IEEE Transactions on Signal Processing, pp. 1018-1027, vol. 56, 2008.

[12] P. H. Bardell, W. H. McAnney, J. Savir, "Built-In Test for VLSI: Pseudorandom Techniques,” John Wiley \& Sons, New York, 1987.

[13] G. A. Franklin, "Using modal analysis to estimate received signals levels for a power-line carrier channel on a 500-kV transmission line", IEEE Transactions on Power Delivery, vol. 45, pp.2446-2454, 2009.

[14] C. A. Janeway, P. Travers, et al., "Immuno Biology," Garland Science, New York, 2005.

[15] K. Tang, G.K. Venayagamoorthy, Online coherency analysis of synchronousgenerators in a power system, in: 2014 IEEE PES Innovative Smart Grid Tech-nologies Conference, 2014.

[16] P. Saraf, G. K. Venayagamoorthy, "Online oscillation monitoring of synchronous generators using parallel-Prony analysis," IEEE PES Innovative Smart Grid Technologies Conference, 2014.

[17] K. Tang, G. K. Venayagamoorthy, “An LMI-SSI model based PSS design approach for a multi-machine power system," 2015 Clemson University Power System Conference, pp. 1-7, 2015.

[18] S. Ray, G. K. Venayagamoorthy, "Real-Time Implementation of a Measurement based Adaptive Wide Area Control System Considering Communication Delays", IET Proceedings on Generation, Transmission and Distribution, Vol. 2, Issue 1, Jan. 2008, pp. 62 - 70.

[19] G. B. Arfken, H. J. Weber, "Mathematical Methods for Physicists,” Harcourt, San Diego, 2001. 